\title{
KEY EXCHANGE OVER PARTICULAR ALGEBRAIC CLOSURE RING
}

\author{
Mohammed Sahmoudi — Abdelhakim Chillali
}

\begin{abstract}
In this paper, we propose a new method of Diffie-Hellman key exchange based on a non-commutative integral closure ring. The key idea of our proposal is that for a given non-commutative ring, we can define the secret key and take it as a common key to encrypt and decrypt the transmitted messages. By doing, we define a new non-commutative structure over the integral closure $O_{L}$ of sextic extension $L$, namely $L$ is an extension of $\mathbb{Q}$ of degree 6 in the form $\mathbb{Q}(\alpha, \beta)$, which is a rational quadratic and monogenic extension over a non-pure and monogenic cubic subfield $K=\mathbb{Q}(\beta)$.
\end{abstract}

\section{Introduction}

The purpose of homomorphic encryption is to allow calculation on encrypted data. Thus, data can remain confidential during treatment, allowing performing useful tasks with data residing in untrusted environments. Finding a general method for computing on encrypted data have been a goal in cryptography since it was proposed in 1978 by Rivest, Adleman and Dertouzos [6]. Principally, fully homomorphic cryptosystems allow for arbitrary computations on encrypted data. Computing on encrypted data means that if a user has a function $\mathrm{f}$ and wants to obtain $f\left(t_{1}, t_{2}, \ldots, t_{n}\right)$ for some $t_{1}, t_{2}, \ldots, t_{n}$ inputs, it is possible to compute on encryptions of these inputs $c_{1}, c_{2}, \ldots, c_{n}$, obtaining a result, which decrypts to $f\left(t_{1}, t_{2}, \ldots, n\right)$. In some cryptosystems, the input messages (plaintexts) lie within some algebraic structure, often a group or a ring as in our work, where the cryptosystem is a homomorphic encryption and decryption scheme. In other terms an encryption function $\operatorname{Enc}()$ such that both $\operatorname{Enc}(\mathrm{x}+\mathrm{y})$ and $\operatorname{Enc}(\mathrm{x} . \mathrm{y})$ are easy to compute from $\operatorname{Enc}(\mathrm{x})$ and $\operatorname{Enc}(\mathrm{y})$. If a user could take a problem defined in one algebraic system and encode it into a problem in a different algebraic system in a way that decoding back to the original

(C) 2017 Mathematical Institute, Slovak Academy of Sciences.

2010 Mathematics Subject Classification: Primary 11T71; Secondary 11Rxx.

Keywords: integral basis, key exchange, fully homomorphic cryptosystems, cryptography.

LAGA Laboratory, Faculty of Sciences Dhar El Mahraz Fèz and FPT in Taza. USMBA. 
algebraic system is hard, then the user could encode expensive computations and send them to the untrusted party. This untrusted party then performs the corresponding computation in the second algebraic system, returning the result to the user. Upon receiving the result, the user can decode it into a solution in the original algebraic system, while the untrusted party learns nothing of which computation is actually performed. Let $\mathrm{L}$ be a sextic number field, namely, $\mathrm{L}$ is an extension of $\mathbb{Q}$ of degree 6 in the form $\mathbb{Q}(\alpha, \beta)$, which is a rational quadratic and monogenic extension over non-pure cubic subfield $K=\mathbb{Q}(\beta)$ see [1], [7. Let $O_{L}$ be the ring of integers of $\mathrm{L}$, Theorem 1 gives a basis of the ring of integer; namely:

$$
O_{L}=\left\{a_{0}+a_{1} \alpha+a_{2} \beta+a_{3} \beta^{2}+a_{4} \alpha \beta+a_{5} \alpha \beta^{2} /\left(a_{i}\right)_{0 \leq i \leq 5} \in \mathbb{Z}^{6}\right\} .
$$

The work presented in this paper comes within the framework of making cryptography on the integral closure of a sextic number field. In [2], we have defined a new multiplicative and commutative structure on $O_{L}$. Here, we define another structure on $O_{L}$, which is not commutative and on which the discrete logarithm problem appears to be much more difficult than in the case discussed in [2].

\section{Integral bases of sextic extension}

In this section, we give an integral basis of sextic field with a non-pure and monogenic cubic subfield, namely $\mathbb{Q}(\alpha, \beta)$, we denote by $O_{L}$ the integral closure of $\mathbb{Z}$ in $\mathrm{L}$.

TheOREM 1. Let $d$ be a square free rational integer and $\alpha$ defined by

$$
\alpha= \begin{cases}\sqrt{d}, & \text { if } d \equiv 2,3 \bmod 4, \\ \frac{1+\sqrt{d}}{2}, & \text { if } d \equiv 1 \bmod 4 .\end{cases}
$$

Let $K=\mathbb{Q}(\beta)$ be a non-pure and monogenic cubic field, where $\beta$ is a root of a monic irreducible polynomial $T(X)=X^{3}-a X+b \in \mathbb{Z}[X]$. Let $L=\mathbb{Q}(\alpha, \beta)$ be a pure quadratic extension of $K$, where $\alpha$ is a root of a monic irreducible polynomial $P(X)=X^{2}-d \in O_{K}$. Suppose that the $p$-adic valuation $v_{p}$ of $b$ equals to $1\left(v_{p}(b)=1\right)$, for all prime integer $p$ in $\mathbb{Z}$. Then the sextic field $L=\mathbb{Q}(\alpha, \beta)$ has an integral basis given by

$$
\mathfrak{B}=\left\{1, \alpha, \beta, \beta^{2}, \alpha \beta, \alpha \beta^{2}\right\} .
$$

P r o of. Indeed $B=\left\{1, \beta, \beta^{2}\right\}$ is an integral basis of $\mathrm{K}$ by [1, Theorem 5.1.], therefore we use [7, Lemma 3.2] and [1, Theorem 1.1]. 


\section{KEY EXCHANGE OVER PARTICULAR ALGEBRAIC CLOSURE RING}

\section{Structure on the ring $L$}

Let $d$ be a square free rational integer and $\beta$ is a root of a monic polynomial $Q(X)=X^{2}-d$. Let $a$ be a rational square free integer, $K$ be the field $\mathbb{Q}(\beta)$, where $\beta$ is a root of $P(X)=X^{3}-a X+b$ and putting $L=\mathbb{Q}(\alpha, \beta)$. By 1 , $\mathfrak{B}=\left\{1, \alpha, \beta, \beta^{2}, \alpha \beta, \alpha \beta^{2}\right\}$ is a basis of $L$ over $\mathbb{Q}$. Let $X, Y \in L$ given by:

$$
\begin{aligned}
& X=x_{0}+x_{1} \alpha+x_{2} \beta+x_{3} \beta^{2}+x_{4} \alpha \beta+x_{5} \alpha \beta^{2}, \\
& Y=y_{0}+y_{1} \alpha+y_{2} \beta+y_{3} \beta^{2}+y_{4} \alpha \beta+y_{5} \alpha \beta^{2},
\end{aligned}
$$

where $\left(x_{0}, x_{1}, x_{2}, x_{3}, x_{4}, x_{5}, y_{0}, y_{1}, y_{2}, y_{3}, y_{4}, y_{5}\right) \in \mathbb{Q}^{12}$. We define over L the following structure:

$$
\begin{gathered}
X+Y=s_{0}+s_{1} \alpha+s_{2} \beta+s_{3} \beta^{2}+s_{4} \alpha \beta+s_{5} \alpha \beta^{2}, \\
X . Y=p_{0}+p_{1} \alpha+p_{2} \beta+p_{3} \beta^{2}+p_{4} \alpha \beta+p_{5} \alpha \beta^{2},
\end{gathered}
$$

where

$$
\begin{aligned}
s_{i} & =x_{i}+y_{i}, \quad \text { for all } i \in\{0,1,2,3,4,5\}, \\
p_{0} & =x_{0} y_{0} \\
p_{1} & =x_{0} y_{1}+x_{1} y_{3} \\
p_{2} & =x_{0} y_{2}+x_{1} y_{4}+x_{2} y_{5}, \\
p_{3} & =x_{3} y_{3} \\
p_{4} & =x_{3} y_{4}+x_{4} y_{5} \\
p_{5} & =x_{5} y_{5} .
\end{aligned}
$$

Theorem 2. The product (.) is an internal composition law on L. Moreover $(L,+,$.$) is a non-commutative and associative ring.$

P r o of. The product is non-commutative, comes from $p_{1}=x_{0} y_{1}+x_{1} y_{3}$, which is clearly not symmetric. For the second statement we put $Z:=z_{0}+z_{1} \alpha+z_{2} \beta+$ $z_{3} \beta^{2}+z_{4} \alpha \beta+z_{5} \alpha \beta^{2}$. Then

$$
(X . Y) . Z=t_{0}+t_{1} \alpha+t_{2} \beta+t_{3} \beta^{2}+t_{4} \alpha \beta+t_{5} \alpha \beta^{2},
$$

where

$$
\begin{aligned}
& t_{0}=x_{0} y_{0} z_{0}, \\
& t_{1}=x_{0} y_{0} z_{1}+x_{0} y_{1} z_{3}+x_{1} y_{3} z_{3}, \\
& t_{2}=x_{0} y_{0} z_{2}+x_{0} y_{1} z_{4}+x_{1} y_{3} z_{4}+x_{0} y_{2} z_{5}+x_{1} y_{4} z_{5}+x_{2} y_{5} z_{5}, \\
& t_{3}=x_{3} y_{3} z_{3}, \\
& t_{4}=x_{3} y_{3} z_{4}+x_{3} y_{4} z_{5}+x_{4} y_{5} z_{5}, \\
& t_{5}=x_{5} y_{5} z_{5} .
\end{aligned}
$$




\section{MOHAMMED SAHMOUDI - ABDELHAKIM CHILLALI}

With the same way we compute $X .(Y . Z)$, we find the same coefficients as in $(X . Y) . Z$. To conclude this proof, we show the distributivity on the left and on the right. $X, Y$ and $Z$ are as above. Using a computing package, such as (Maple), we check that:

$$
\begin{gathered}
(Y+Z) . X=h_{0}+h_{1} \alpha+h_{2} \beta+h_{3} \beta^{2}+h_{4} \alpha \beta+h_{5} \alpha \beta^{2}, \\
Y . X+Z . X=g_{0}+g_{1} \alpha+g_{2} \beta+g_{3} \beta^{2}+g_{4} \alpha \beta+g_{5} \alpha \beta^{2}
\end{gathered}
$$

with

$$
\begin{aligned}
& h_{0}=x_{0}\left(y_{0}+z_{0}\right), \\
& h_{1}=\left(y_{1}+z_{1}\right) x_{0}+\left(y_{3}+z_{3}\right) x_{1}, \\
& h_{2}=x_{0}\left(y_{2}+z_{2}\right)+x_{1}\left(y_{4}+z_{4}\right)+x_{2}\left(y_{5}+z_{5}\right), \\
& h_{3}=x_{3}\left(y_{3}+z_{3}\right), \\
& h_{4}=x_{3}\left(y_{4}+z_{4}\right)+x_{4}\left(y_{5}+z_{5}\right), \\
& h_{5}=x_{5}\left(y_{5}+z_{5}\right),
\end{aligned}
$$

and

$$
\begin{aligned}
& g_{0}=x_{0} y_{0}+x_{0} z_{0}, \\
& g_{1}=x_{0} y_{1}+x_{1} y_{3}+x_{0} z_{1}+x_{1} z_{3}, \\
& g_{2}=x_{0} y_{2}+x_{1} y_{4}+x_{2} y_{5}+x_{0} z_{2}+x_{1} z_{4}+x_{2} z_{5}, \\
& g_{3}=x_{3} y_{3}+x_{3} z_{3}, \\
& g_{4}=x_{3} y_{4}+x_{4} y_{5}+x_{3} z_{4}+x_{4} z_{5}, \\
& g_{5}=x_{5} y_{5}+x_{5} z_{5} .
\end{aligned}
$$

It's clear that for all $i$ in $\{0, \ldots, 5\} ; h_{i}=g_{i}$.

Proposition 1. Let $X \in L$ given by: $X=x_{0}+x_{1} \alpha+x_{2} \beta+x_{3} \beta^{2}+x_{4} \alpha \beta+x_{5} \alpha \beta^{2}$. The neutral element for the group law $(L,$.$) is the point e equal to 1+\beta^{2}+\alpha \beta^{2}$. On the other hand, $X$ is invertible in $L$ if and only if $x_{0} x_{3} x_{5} \neq 0$. Indeed, the inverse $Y$ is

$$
Y=i_{0}+i_{1} \alpha+i_{2} \beta+i_{3} \beta^{2}+i_{4} \alpha \beta+i_{5} \alpha \beta^{2},
$$

where

$$
\begin{aligned}
i_{0} & =\frac{1}{x_{0}} ; & i_{1} & =-\frac{x_{1}}{x_{0} x_{3}} ; \\
i_{2} & =\frac{x_{1} x_{4}-x_{2} x_{3}}{x_{0} x_{3} x_{5}} ; & i_{3} & =\frac{1}{x_{3}} ; \\
i_{4} & =\frac{-x_{4}}{x_{5} x_{3}} ; & i_{5} & =\frac{1}{x_{5}} .
\end{aligned}
$$




\section{KEY EXCHANGE OVER PARTICULAR ALGEBRAIC CLOSURE RING}

P r o of. It is simple, but less natural, to check directly with the given formulas of unit element e that for all $X \in L, X . e=e \cdot X=X$. Using the product law defined one finds immediately that the inverse of $X$ is equal to $Y$.

Theorem 3. Let $p$ be a positive integer. Then if $X$ is any element of $L: X=$ $x_{0}+x_{1} \alpha+x_{2} \beta+x_{3} \beta^{2}+x_{4} \alpha \beta+x_{5} \alpha \beta^{2}$. We give the $p-$ power of $X$ by: $X^{n}=$ $p_{0}+p_{1} \alpha+p_{2} \beta+p_{3} \beta^{2}+p_{4} \alpha \beta+p_{5} \alpha \beta^{2}$, where

$$
\begin{aligned}
& p_{0}=x_{0}^{n}, \\
& p_{1}=x_{1} \sum_{i+j=n-1} x_{0}^{i} x_{3}^{j}, \\
& p_{2}=x_{2} \sum_{i+j=n-1} x_{0}^{i} x_{5}^{j}+x_{1} x_{4} \sum_{i+j+k=n-2} x_{0}^{i} x_{5}^{j} x_{3}^{k}, \\
& p_{3}=x_{3}^{n}, \\
& p_{4}=x_{4} \sum_{0 \leq i \leq n-1} x_{3}^{i} x_{5}^{n-1-i}, \\
& p_{5}=x_{5}^{n} .
\end{aligned}
$$

Pr o of. By induction on $\mathrm{n}$. For $n=1$ : the right-hand side in 10 holds since the set $S=\left\{(i, j, k) \in \mathbb{N}^{3} ; i+j+k=-1\right\}$ is empty.

Assume that the formula holds for $n=p$; we will prove it for $n=p+1$. Let $n=p$. Then

$$
\begin{aligned}
& p_{0}=x_{0}^{p}, \\
& p_{1}=x_{1} \sum_{i+j=p-1} x_{0}^{i} x_{3}^{j}, \\
& p_{2}=x_{2} \sum_{i+j=p-1} x_{0}^{i} x_{5}^{j}+x_{1} x_{4} \sum_{i+j+k=p-2} x_{0}^{i} x_{5}^{j} x_{3}^{k}, \\
& p_{3}=x_{3}^{p}, \\
& p_{4}=x_{4} \sum_{0 \leq i \leq p-1} x_{3}^{i} x_{5}^{p-1-i}, \\
& p_{5}=x_{5}^{p} .
\end{aligned}
$$

Show that 11 holds for $n=p+1$, we start with

$$
X^{p+1}=X^{p} \cdot X=q_{0}+q_{1} \alpha+q_{2} \beta+q_{3} \beta^{2}+q_{4} \alpha \beta+q_{5} \alpha \beta^{2} .
$$




\section{MOHAMMED SAHMOUDI - ABDELHAKIM CHILLALI}

Then by the formula 4;

$$
\begin{aligned}
q_{0}= & x_{0}^{p} x_{0}=x_{0}^{p+1}, \\
q_{1}= & p_{0} x_{1}+p_{1} x_{3}=x_{0}^{p} x_{1}+x_{1} x_{3} \sum_{i+j=p-1} x_{0}^{i} x_{3}^{j}, \\
q_{2}= & p_{0} x_{2}+p_{1} x_{4}+p_{2} x_{5}=x_{0}^{p} x_{2}+x_{4} x_{1} \sum_{i+j=p-1} x_{0}^{i} x_{3}^{j} \\
& +\left[x_{2} \sum_{i+j=p-1} x_{0}^{i} x_{5}^{j}+x_{1} x_{4} \sum_{i+j+k=p-2} x_{0}^{i} x_{5}^{j} x_{3}^{k}\right] x_{5}, \\
q_{3}= & x_{3}^{p} x_{3}=x_{3}^{p+1}, \\
q_{4}= & p_{3} x_{4}+p_{4} x_{5}=x_{3}^{p} x_{4}+x_{5} x_{4} \sum_{0 \leq i \leq p-1} x_{3}^{i} x_{5}^{p-i}, \\
q_{5}= & x_{5}^{p} x_{5}=x_{5}^{p+1} .
\end{aligned}
$$

By reindexation, we find:

$$
\begin{aligned}
q_{0}= & x_{0}^{p} x_{0}=x_{0}^{p+1}, \\
q_{1}= & x_{1}\left(x_{0}^{p}+\sum_{i+j=p-1} x_{0}^{i} x_{3}^{j+1}\right)=x_{1} \sum_{i+j^{\prime}=p} x_{0}^{i} x_{3}^{j^{\prime}}\left(j^{\prime}=j-1\right) \\
q_{2}= & x_{0}^{p} x_{2}+x_{4} x_{1} \sum_{i+j=p-1} x_{0}^{i} x_{3}^{j}+x_{2} \sum_{i+j=p-1} x_{0}^{i} x_{5}^{j}+x_{1} x_{4} \sum_{i+j+k=p-2} x_{0}^{i} x_{5}^{j+1} x_{3}^{k} \\
= & x_{0}^{p} x_{2}+x_{2} \sum_{i+j=p-1} x_{0}^{i} x_{5}^{j}+x_{4} x_{1} \sum_{i+j+k=p-1, j=0} x_{0}^{i} x_{5}^{j} x_{3}^{k} \\
& +x_{4} x_{1} \sum_{i+j+k=p-1, j \geq 1} x_{0}^{i} x_{5}^{j} x_{3}^{k} x_{0}^{p} x_{2}+x_{2} \sum_{i+(j+1)=p} x_{0}^{i} x_{5}^{j}+x_{4} x_{1} \sum_{i+j+k=p-1, j=0} x_{0}^{i} x_{5}^{j} x_{3}^{k} \\
& +x_{4} x_{1} \sum_{i+j^{\prime}+k=p-2, j \geq 1} x_{0}^{i} x_{5}^{j^{\prime}} x_{3}^{k} \\
= & x_{0}^{p} x_{2}+x_{2} \sum_{i+j^{\prime \prime}=p} x_{0}^{i} x_{5}^{j^{\prime \prime}}+x_{4} x_{1} \sum_{i+j+k=p-1} x_{0}^{i} x_{5}^{j} x_{3}^{k}\left(j^{\prime \prime}=j+1\right) \\
= & x_{2} \sum_{i+j^{\prime \prime}=p} x_{0}^{i} x_{5}^{j^{\prime \prime}}+x_{4} x_{1} \sum_{i+j+k=p-1} x_{0}^{i} x_{5}^{j} x_{3}^{k},
\end{aligned}
$$




$$
\begin{aligned}
q_{3} & =x_{3}^{p} x_{3}=x_{3}^{p+1}, \\
q_{4} & =p_{3} x_{4}+p_{4} x_{5}=x_{4}\left(x_{3}^{p}+x_{5} \sum_{0 \leq i \leq p-1} x_{3}^{i} x_{5}^{p-1-i}\right) \\
& =x_{4}\left(x_{3}^{p}+\sum_{0 \leq i \leq p-1} x_{3}^{i} x_{5}^{p-i}\right)=x_{4} \sum_{0 \leq i \leq p} x_{3}^{i} x_{5}^{p-i}, \\
q_{5} & =x_{5}^{p} x_{5}=x_{5}^{p+1} .
\end{aligned}
$$

\section{Key exchange on $O_{L}$}

\subsection{Key exchange}

Key exchange is a method of securely exchanging cryptographic keys over a public channel [4], [8]. The Diffie-Hellman key exchange is the following protocol:

- Alice and Bob choose a common element $\left(x_{0}, x_{3}, x_{5}\right) \in \mathbb{Z}^{3}$.

- Alice chooses an element $X=x_{0}+x_{1} \alpha+x_{2} \beta+x_{3} \beta^{2}+x_{4} \alpha \beta+x_{5} \alpha \beta^{2} \in O_{L}$.

- Bob chooses an element $Y=x_{0}+y_{1} \alpha+y_{2} \beta+x_{3} \beta^{2}+y_{4} \alpha \beta+x_{5} \alpha \beta^{2} \in O_{L}$.

- Alice chooses an integer $n$. First, she computes $X^{n}=f_{0}+f_{1} \alpha+f_{2} \beta+$ $f_{3} \beta^{2}+f_{4} \alpha \beta+f_{5} \alpha \beta^{2}$. After, she transmits the new element denoted by $X_{a}$ to Bob, namely, $X_{a}=t_{0}+t_{1} \alpha+t_{2} \beta+t_{3} \beta^{2}+t_{4} \alpha \beta+t_{5} \alpha \beta^{2}$ with $t_{0}=x_{0}, t_{3}=x_{3}, t_{5}=x_{5} \cdot t_{1}=f_{1}, t_{2}=f_{2}, t_{4}=f_{4}$.

- Similarly, Bob chooses an integer $m$ and computes $Y^{m}=g_{0}+g_{1} \alpha+g_{2} \beta+$ $g_{3} \beta^{2}+g_{4} \alpha \beta+g_{5} \alpha \beta^{2}$. Once calculated, Bob transmits the new element $Y_{b}$ to Alice where $Y_{b}=\delta_{0}+\delta_{1} \alpha+\delta_{2} \beta+\delta_{3} \beta^{2}+\delta_{4} \alpha \beta+\delta_{5} \alpha \beta^{2}$ with $\delta_{0}=x_{0}$, $\delta_{3}=x_{3}, \delta_{5}=x_{5} \cdot \delta_{1}=g_{1}, \delta_{2}=g_{2}, \delta_{4}=g_{4}$.

- Alice computes $Y_{b}^{n}=d_{0}+d_{1} \alpha+d_{2} \beta+d_{3} \beta^{2}+d_{4} \alpha \beta+d_{5} \alpha \beta^{2}$.

- Bob computes $X_{a}^{m}=r_{0}+r_{1} \alpha+r_{2} \beta+r_{3} \beta^{2}+r_{4} \alpha \beta+r_{5} \alpha \beta^{2}$.

Now to give the secret key, we need to create links between $d_{i}$ and $r_{i}$.

LEMMA 1. We have $x_{1} d_{1}=y_{1} r_{1}$ and $x_{4} d_{4}=y_{4} r_{4}$, which will be denoted $k_{1}$ and $k_{2}$, respectively.

P r o of. According to the power formulas, we have:

$$
d_{1}=y_{1} \sum_{i+j=n-1} x_{0}^{i} x_{3}^{j}, \quad r_{1}=x_{1} \sum_{i+j=n-1} x_{0}^{i} x_{3}^{j} .
$$

Then, $x_{1} d_{1}=y_{1} r_{1}$. 
Of the same one finds,

$$
d_{4}=y_{4} \sum_{i+j=n-1} x_{0}^{i} x_{3}^{j}, \quad r_{4}=x_{4} \sum_{i+j=n-1} x_{0}^{i} x_{3}^{j}
$$

which shows $x_{4} d_{4}=y_{4} r_{4}$. The common secret key

$$
k=1+k_{1} \alpha+k_{2} \beta+\beta^{2}+k_{4} \alpha \beta+\alpha \beta^{2}
$$

with

$$
k_{1}=x_{1} d_{1}, \quad k_{2}=x_{4} d_{4}, \quad \text { and } \quad k_{4}=\text { next prime }\left(k_{1} \cdot k_{2}\right)
$$

Problem(*). Let $X$ and $X_{a}$ in $O_{L}$, find the integer $n$ such that

$$
\left\{\begin{array}{l}
t_{1}=x_{1} \sum_{i+j=n-1} x_{0}^{i} x_{3}^{j}, \\
t_{2}=x_{2} \sum_{i+j=n-1} x_{0}^{i} x_{5}^{j}+x_{1} x_{4} \sum_{i+j+k=n-2} x_{0}^{i} x_{5}^{j} x_{3}^{k}, \\
t_{4}=x_{4} \sum_{0 \leq i \leq n-1} x_{3}^{i} x_{5}^{n-i} .
\end{array}\right.
$$

Assumption. There is no polynomial or sub-exponential algorithm that can calculate the integer $n$ in the previous problem.

\subsection{Function of encryption - function of decryption}

Before defining the functions of encryption and decryption, we will introduce some spaces, which will be useful to us later:

- Space of lights: $M=O_{L}$.

- Space of quantified: $C=O_{L}$.

- Space of the keys: $K=U\left(O_{L}\right)$; unit of $O_{L}$.

\subsubsection{Function of encryption}

For all $k$ in $K$, the function of encryption is defined by:

$$
\begin{aligned}
e_{k}: M & \rightarrow C, \\
m & \mapsto k \cdot m . k^{-1} .
\end{aligned}
$$




\section{KEY EXCHANGE OVER PARTICULAR ALGEBRAIC CLOSURE RING}

\subsubsection{Function of decryption}

For all $k$ in $K$, the function of decryption is defined by:

$$
\begin{aligned}
d_{k}: C & \rightarrow M, \\
c & \mapsto k^{-1} . c . k .
\end{aligned}
$$

Remark 1. The functions $e_{k}$ and $d_{k}$ are well defined since $k$ admits an inverse in $K$, consequently, we can check immediately that: $d_{k} \circ e_{k}(m)=m$, for all $m \in M$.

Remark 2. The algebraic integer $e_{k}(m)$ is public and is known to other persons, but it is necessary to solve the problem $(*)$ to obtain the secret key $k$.

Lemma 2. Let $\left(m_{1}, m_{2}\right) \in M^{2}$. Then:

- $e_{k}\left(m_{1}+m_{2}\right)=e_{k}\left(m_{1}\right)+e_{k}\left(m_{2}\right)$,

- $e_{k}\left(m_{1} \cdot m_{2}\right)=e_{k}\left(m_{1}\right) \cdot e_{k}\left(m_{2}\right)$.

Proof.

On one hand:

- $e_{k}\left(m_{1}+m_{2}\right)=k \cdot\left(m_{1}+m_{2}\right) \cdot k^{-1}=k \cdot m_{1} \cdot k^{-1}+k \cdot m_{2} \cdot k^{-1}$ $=e_{k}\left(m_{1}\right)+e_{k}\left(m_{2}\right)$.

On the other hand:

- $e_{k}\left(m_{1} \cdot m_{2}\right)=k \cdot\left(m_{1} \cdot m_{2}\right) \cdot k^{-1}=\left(k \cdot m_{1} \cdot k^{-1}\right) \cdot\left(k \cdot m_{2} \cdot k^{-1}\right)$ $=e_{k}\left(m_{1}\right) \cdot e_{k}\left(m_{2}\right)$.

Remark 3. This cryptosystem is a homomorphic encryption and decryption scheme.

\section{Numerical example}

In this section, we will try to give a numerical example of the cryptosystem thus constructed. For that, we put, $n=461, m=562$ and we give a prime number $p=46613216676546898945626221516$. Alice and Bob choose two algebraic integers $X, Y$ transformed as a list:

$X=[54641626461316643235655555555555555556514123232355$, 5546554421665698989845798995656465621998987985979959,2545499621

$5465512115454462124545,51545454455545412124545151545151151,6$ 51445998988888888888888888888865656,654465674698898977979979 71299999999991] $\bmod p$. 
$Y=[54641626461316643235655555555555555556514123232355,55446$ 55442165621998987985979959,254554996215465588787458488787871 $2115454462124545,51545454455545412124545151545151151,65888889$ 98941551894465161545488888888888865656,654465674698898977979 97971299999999991] $\bmod p$.

$X_{a}=54641626461316643235655555555555555556514123232355$, 1242114200316417343556537318459, 2758940433977467632778875714860, 51545454455545412124545151545151151,1353358853755699392084137323774 , $65446567469889897797997971299999999991 \bmod p$.

$Y_{b}=54641626461316643235655555555555555556514123232355,18437$ $04017016984727977634474812,422284753902375546076271379645,515$ 45454455545412124545151545151151,8818760132292706792594977964 $76,65446567469889897797997971299999999991 \bmod p$.

$Y_{b}^{n}=[2169497613847660334199711549428,1506827088352661952016$ $372541470,2861039411877654648157728023978,3132620795697456678$ 692624481121, 967703610901502040900632958300,18403999185170136 76297144748199] $\bmod p$.

$X_{a}^{m}=[2169497613847660334199711549428,5658005008936889441365$ 62778407, 2722703841178032092187769200383, 31326207956974566786 $92624481121,3415551958588233675081576426158,18403999185170136$ 76297144748199] $\bmod p$.

The common secret key:

$$
k=1+k_{1} \alpha+k_{2} \beta+\beta^{2}+k_{4} \alpha \beta+\alpha \beta^{2}
$$

with

- $k_{1}=2733283161771536899835743622228$,

- $k_{2}=2220752190347668348403326022796$,

- $k_{4}=3810658765422065314614619743114$.

The inverse of the common secret key:

$$
k^{-1}=1+k_{11} \alpha+k_{21} \beta+\beta^{2}+k_{41} \alpha \beta+\alpha \beta^{2}
$$

with

- $k_{11}=1928038505883152994726878529419$,

- $k_{21}=3399036551396573130220673517652$,

- $k_{41}=850662902232624579948002408533$. 


\section{KEY EXCHANGE OVER PARTICULAR ALGEBRAIC CLOSURE RING}

The encryption message:

$$
\begin{aligned}
c= & e_{k}(X \bmod p)=k \cdot m \cdot k^{-1} \\
= & 48385458079557240049145558524+284643977367852885483899 \\
& 0945055 . \alpha+63273121327215098070824065451 \cdot \beta-59454 \\
& 619851270471675792238625 . \beta^{2}-16204005879189078877631 \\
& 34802282 . \alpha \beta+3100042710810325132186233801776 . \alpha \beta^{2}
\end{aligned}
$$

The decryption message:

$$
\begin{aligned}
m= & d_{k}(c) \\
= & {[54641626461316643235655555555555555556514123232355,} \\
& 5546554421665698989845798995656465621998987985979959, \\
& 25454996215465512115454462124545,51545454455545412124 \\
& 545151545151151,651445998988888888888888888888865656, \\
& 65446567469889897797997971299999999991] \bmod p .
\end{aligned}
$$

Conclusion 1. In conclusion, we have presented a key exchange on the infinity ring $O_{L}$, which is based on the discrete logarithm problem. To give an example of cryptography, we can build a set called generic set over $O_{L}$ on which the cryptosystem whose secret key $K$ has stemmed from Diffie-Hellman Key Exchange on $O_{L}$. This idea is the object of a next paper.

\section{Open problem}

Let $X$ and $X_{a}$ in $O_{L}$ as defined above in the beginning of the fourth section. Is it easy to find the integer $n$ such that

$$
\left\{\begin{array}{l}
t_{1}=x_{1} \sum_{i+j=n-1} x_{0}^{i} x_{3}^{j}, \\
t_{2}=x_{2} \sum_{i+j=n-1} x_{0}^{i} x_{5}^{j}+x_{1} x_{4} \sum_{i+j+k=n-2} x_{0}^{i} x_{5}^{j} x_{3}^{k}, \\
t_{4}=x_{4} \sum_{0 \leq i \leq n-1} x_{3}^{i} x_{5}^{n-i} .
\end{array}\right.
$$

Can we generalize this work in an upper degree for the extension $L$, knowing that the difficulty of the problem becomes more difficult every time the degree of the extension becomes bigger? 
Acknowledgements. The authors would like to thank LAGA Laboratory, Faculty of Sciences Dhar El Mahraz Fez and FPT in Taza. USMBA, MOROCCO for its valued support.

\title{
REFERENCES
}

[1] CHARKANI, M. E.-SAHMOUDI, M.: Sextic extension with cubic subfield, JP J. Algebra, Number Theory Appl. 34 (2014), 139-150.

[2] CHILlAli, A.-SAHMOUDI, M.: Cryptography over sextic extension with cubic subfield, World Academy Sci. Engrg. Technol. 9 (2015), 246-249.

[3] COHEN, H.: A Course in Computational Algebraic Number theory. GTM Springer-Verlag, Berlin, 1996.

[4] DIFFIE, W.-HELLMAN, M. E.: New directions in cryptography, IEEE Trans. Inf. Theory 22 (1976), 644-654.

[5] MEnEZES, A. J.: Elliptic Curve Public key Cryptosystems. Foreword by Neal Koblitz. In: Kluwer Internat. Ser. Engrg. Comput. Sci., Vol. 234, Kluwer Acad. Publ., Boston, MA, 1993.

[6] RIVEST, R. L.-ADLEMAN, L.-DERTOUZOS, M. L.: On data banks and privacy homomorphisms. Foundations of Secure Computation 11, (1978) no. 4, 169-180.

[7] SAHMOUDI, M.: Explicit integral basis for a family of sextic field, Gulf J. Math. 4 (2016), $217-222$.

[8] MILLER, V. S.: Use of elliptic curves in cryptography. In: Advances in Cryptology-CRYPTO '85 (H. C. Williams, ed.), Proc. Conf., Santa Barbara/Calif. 1985, Lect. Notes Comput. Sci., Vol. 218, Springer, Berlin, 1986, pp. 417-426.

Received March, 25, 2017

\author{
Mohammed Sahmoudi \\ LAGA Laboratory \\ Faculty of Sciences Dhar El Mahraz \\ P. 0. Box 1796, Atlas-Fez \\ MOROCCO \\ E-mail: mohamed-sahmoudi@usmba.ac.ma \\ Abdelhakim Chillali \\ Sidi Mohamed Ben Abdellah University \\ FP, LSI, Taza, \\ MOROCCO \\ E-mail: abdelhakim.chillali@usmba.ac.ma
}

